\title{
Supplementary Information: Understanding the Electron-Doping Mechanism in Potassium-Intercalated Single-Walled Carbon Nanotubes
}

\author{
Claudia Kröckel, ${ }^{1}$ María Rosa Preciado-Rivas, ${ }^{2}$ Victor Alexander Torres-Sánchez, ${ }^{2}$ Duncan J. \\ Mowbray, ${ }^{2}$ Stephanie Reich, ${ }^{3}$ Frank Hauke, ${ }^{1}$ Julio C. Chacón-Torres, ${ }^{2}$ and Andreas Hirsch ${ }^{1}$ \\ ${ }^{1}$ Department of Chemistry and Pharmacy and Joint Institute of Advanced Materials and Processes (ZMP), Friedrich- \\ Alexander University of Erlangen-Nuremberg, Nikolaus-Fiebiger-Str. 10, 91058 Erlangen, Germany \\ ${ }^{2}$ Yachay Tech University, School of Physical Sciences and Nanotechnology, 100119-Urcuquí, Ecuador \\ ${ }^{3}$ Department of Physics, Freie Universität Berlin, Arnimallee 14, 14195 Berlin, Germany
}

\section{RBM fitting procedure}

All RBM peaks were fitted with Lorentz functions. We found a linear function to describe the background best in the metallic enriched sample. In the semiconducting enriched sample we had to use a quadratic function for the background due to the large fluorescent effect getting prominent at an increasing doping level. We found that the FWHM was not changed in the semiconducting species with doping. Thus, we kept the FWHM fixed for the semiconducting chiralities for all fittings in both samples. The FWHM was also kept fixed for the metallic species in the metallic enriched sample until minute 16. Afterwards accurate fits were just possible with keeping the FWHM free for the metallic species. In the semiconducting enriched sample the FWHM had to kept free for the metallic species in all fittings. We assigned this to the low amount of metallic species $(<2 \%)$ in the semiconducting enriched sample what could also be seen in the low signal intensity. Thus, the metallic tubes were affected fast by the negative charges leading to inaccurate fits with a fixed FWHM. 


\section{Pristine spectra of the metallic and semiconducting enriched HiPco SWCNTs}

(a) HiPco metallic

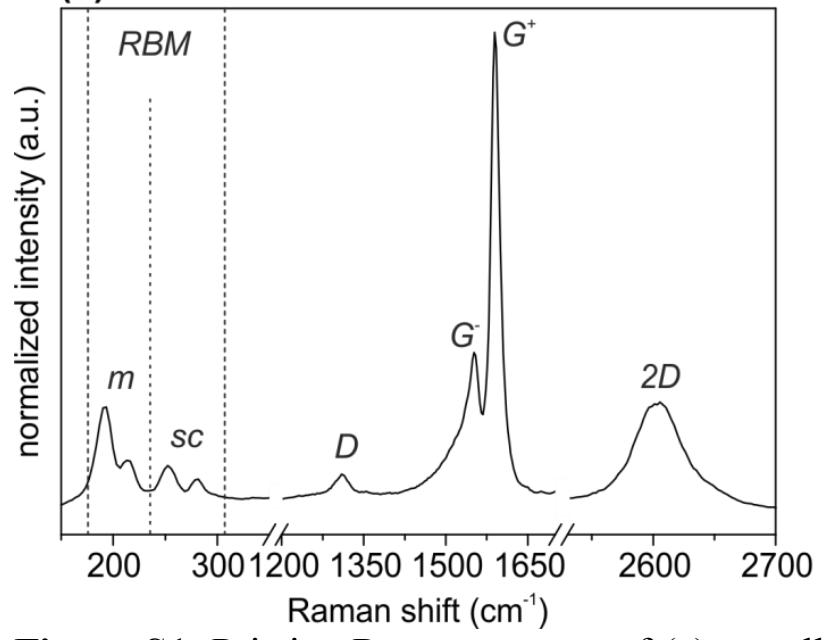

(b) HiPco semiconducting

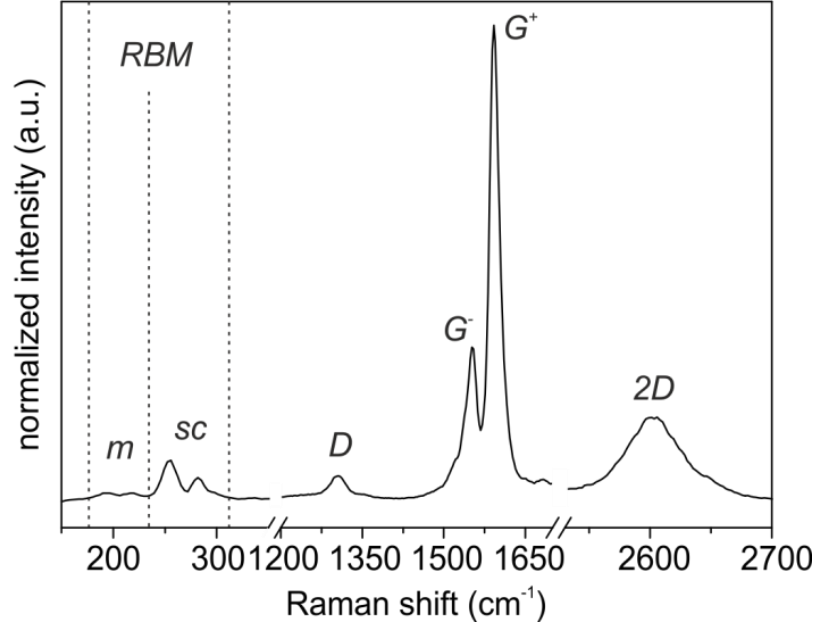

Figure S1. Pristine Raman spectra of (a) metallic and (b) semiconducting enriched SWCNTs at an excitation wavelength of $633 \mathrm{~nm}$. 


\section{Calculated chiralities}

\begin{tabular}{|c|c|c|c|c|c|}
\hline$\omega_{R B M}^{f i t}\left(\mathrm{~cm}^{-1}\right)$ & $\boldsymbol{d}_{\boldsymbol{c a l c}}(\mathrm{nm})$ & $\begin{array}{l}\text { assigned } \\
\text { chirality }\end{array}$ & $\boldsymbol{d}_{\text {theor }}(\mathrm{nm})^{1}$ & chiral angle $\theta^{1}$ & $\boldsymbol{\omega}_{\boldsymbol{R} \boldsymbol{B} \boldsymbol{M}}^{\boldsymbol{l i t}}\left(\mathrm{cm}^{-1}\right)^{2}$ \\
\hline \multicolumn{6}{|c|}{ metallic enriched sample } \\
\hline 188.9 & 1.258 & $(12,6)$ & 1.244 & 19.1 & $178-195$ \\
\hline \multirow{2}{*}{193.7} & \multirow{2}{*}{1.224} & $(13,4)$ & 1.206 & 13.0 & $183-201$ \\
\hline & & $(9,9)$ & 1.221 & 30.0 & $181-199$ \\
\hline 212.5 & 1.105 & $(11,5)$ & 1.111 & 17.8 & $199-219$ \\
\hline 217.6 & 1.077 & $(12,3)$ & 1.077 & 10.9 & $218^{3}, 217.4^{4}$ \\
\hline 250.8 & 0.924 & $(10,3)$ & 0.924 & 12.7 & $239-263$ \\
\hline \multirow{2}{*}{256.5} & \multirow{2}{*}{0.901} & $(11,1)$ & 0.903 & 4.3 & $245-269$ \\
\hline & & $(7,6)$ & 0.883 & 27.5 & $250-275$ \\
\hline \multirow{2}{*}{281.3} & \multirow{2}{*}{0.817} & $(7,5)$ & 0.818 & 24.5 & $270-297$ \\
\hline & & $(8,4)$ & 0.829 & 19.1 & $267-293$ \\
\hline 292.0 & 0.785 & $(8,3)$ & 0.772 & 15.3 & $286-315$ \\
\hline \multicolumn{6}{|c|}{ semiconducting enriched sample } \\
\hline \multirow{2}{*}{193.7} & \multirow{2}{*}{1.224} & $(13,4)$ & 1.206 & 13.0 & $183-201$ \\
\hline & & $(9,9)$ & 1.221 & 30.0 & $181-199$ \\
\hline 218.2 & 1.074 & $(12,3)$ & 1.077 & 10.9 & $218^{3}, 217.4^{4}$ \\
\hline 250.1 & 0.926 & $(10,3)$ & 0.924 & 12.7 & $239-263$ \\
\hline \multirow{2}{*}{257.2} & \multirow{2}{*}{0.899} & $(11,1)$ & 0.903 & 4.3 & $245-269$ \\
\hline & & $(7,6)$ & 0.883 & 27.5 & $250-275$ \\
\hline \multirow{2}{*}{281.6} & \multirow{2}{*}{0.816} & $(\mathbf{7 , 5})$ & 0.818 & 24.5 & $270-297$ \\
\hline & & $(8,4)$ & 0.829 & 19.1 & $267-293$ \\
\hline 292.0 & 0.785 & $(8,3)$ & 0.772 & 15.3 & $286-315$ \\
\hline
\end{tabular}

Table S1. Calculated diameters and assigned chiralities from the fitted RBM frequencies in the metallic and semiconducting enriched samples extracted from the fitting in Figure 1 (b) and (c). For comparison the theoretical values of the diameters and experimentally obtained RBM regions from literature are listed. The signals at 193, 257 and $281 \mathrm{~cm}^{-1}$ could be caused by caused by two different chiralities, respectively. The ones we have assigned in this case are in bold print. 


\section{Additional steps in the evolution of the Raman spectra with K doping}

(a) HiPco metallic

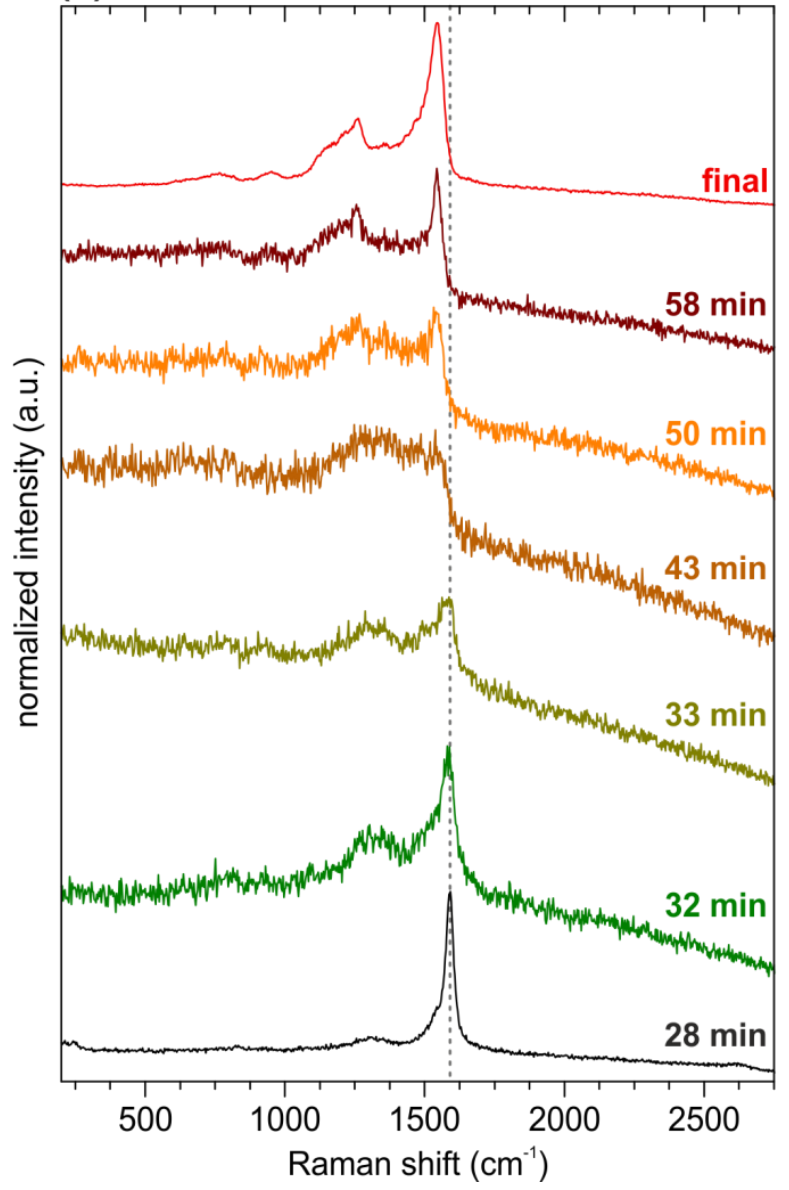

(b) HiPco semiconducting

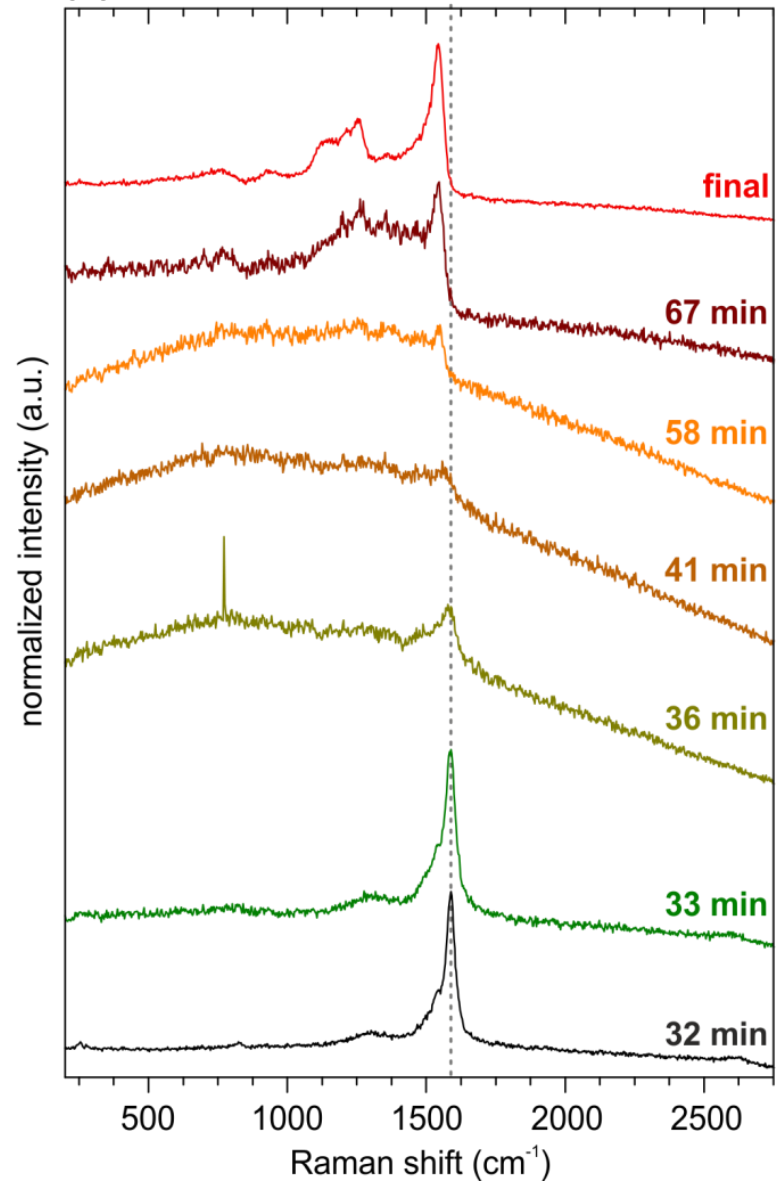

Figure S2. Evolution of the Raman spectra of (a) metallic and (b) semiconducting enriched SWCNTs doped with potassium at different selected doping times recorded with an excitation wavelength of $633 \mathrm{~nm}$. All spectra are normalized to the $\mathrm{G}(+)$ line. The RBMs and the 2D line have completely vanished after $32 \mathrm{~min}$ in the case of the metallic enriched sample and after 36 min in the case of the semiconducting enriched sample. In addition, a clear shift of $\mathrm{G}(+)$ with increasing doping level is visible. 


\section{Absorption spectra of doped HiPCO metallic and semiconducting enriched SWCNTs}

(a) HiPco metallic

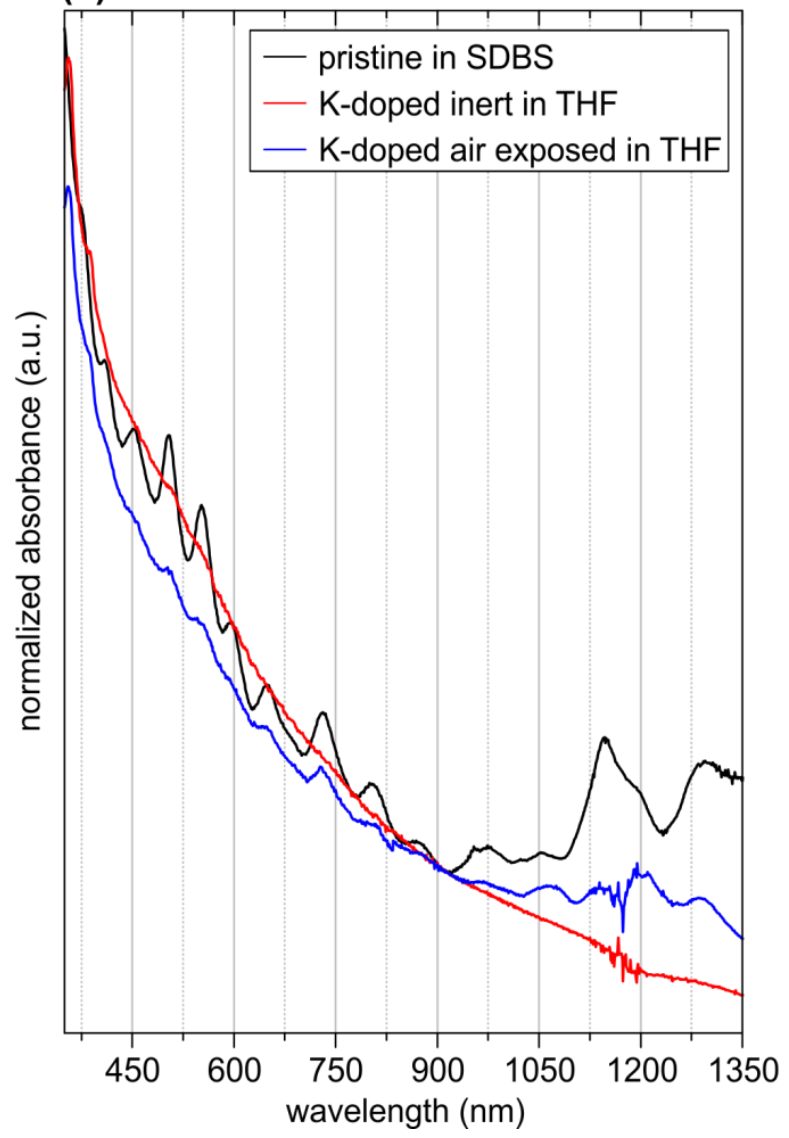

(b) HiPco semiconducting

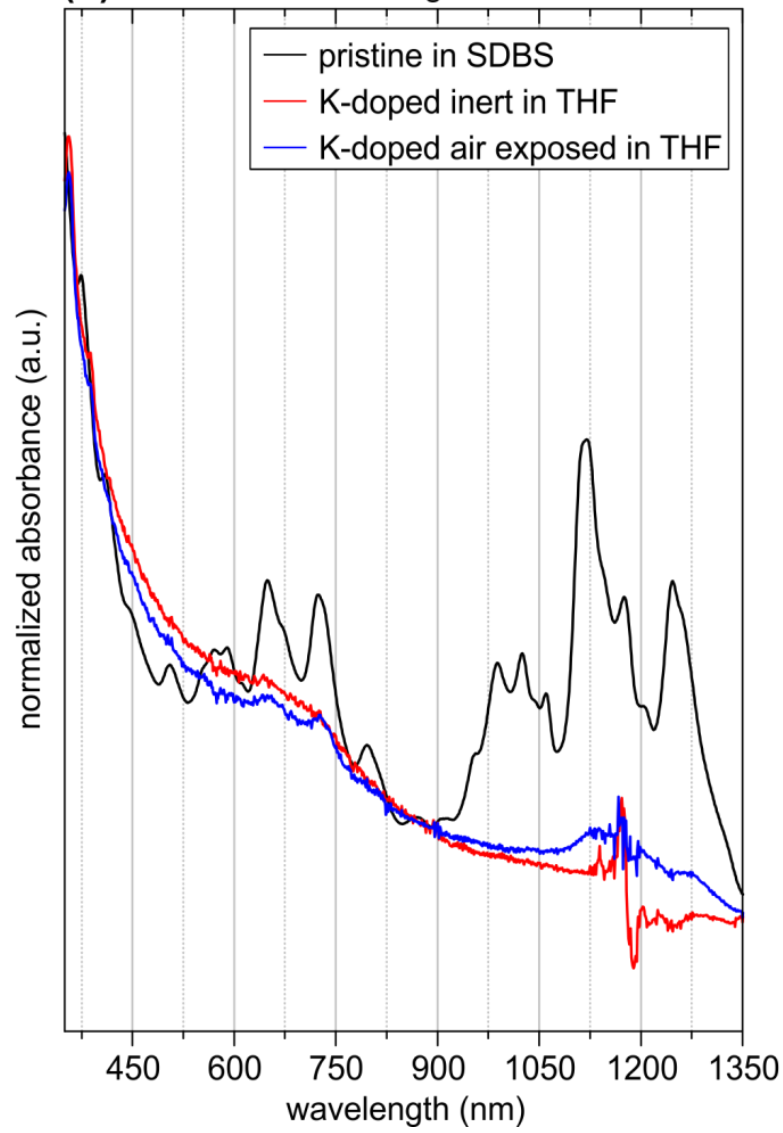

Figure S3. Optical absorption (OA) spectra of HiPco (a) metallic and (b) semiconducting enriched SWCNTs. The black line represent the pristine material dispersed in SDBS (1 wt \%), the red line the K-doped species dispersed in THF in a glovebox under argon atmosphere and the blue line the K-doped species dispersed in THF and opened to ambient conditions.

The pristine samples were measured from a dispersion in SDBS (1 wt \%) since it was not possible to debundle and stabilize neutral SWCNTs with THF to yield a stable dispersion.

The K-doped samples were dispersed via ultrasound in dried and degased THF in a glovebox under argon atmosphere $\left(\mathrm{O}_{2}<0.1 \mathrm{ppm} ; \mathrm{H}_{2} \mathrm{O}<0.1 \mathrm{ppm}\right)$, yielding an almost clear and colorless dispersion for the metallic enriched sample. In the case of the semiconducting enriched tubes the sedimentation of a part of the sample was obtained.

All samples were measured from a sealed cuvette under inert conditions. To ensure the presence of K-doped SWCNTs in the THF dispersion, the cuvette was exposed to air and the samples were measured again under ambient conditions.

All optical absorption spectra were recorded on a Perkiner Elmer Lambda 1050 from precision quartz cuvettes (Hellma, Germany) with a path length of $10 \mathrm{~mm}$. 


\section{RBM shift and intensity development of the semiconducting enriched SWCNTs}
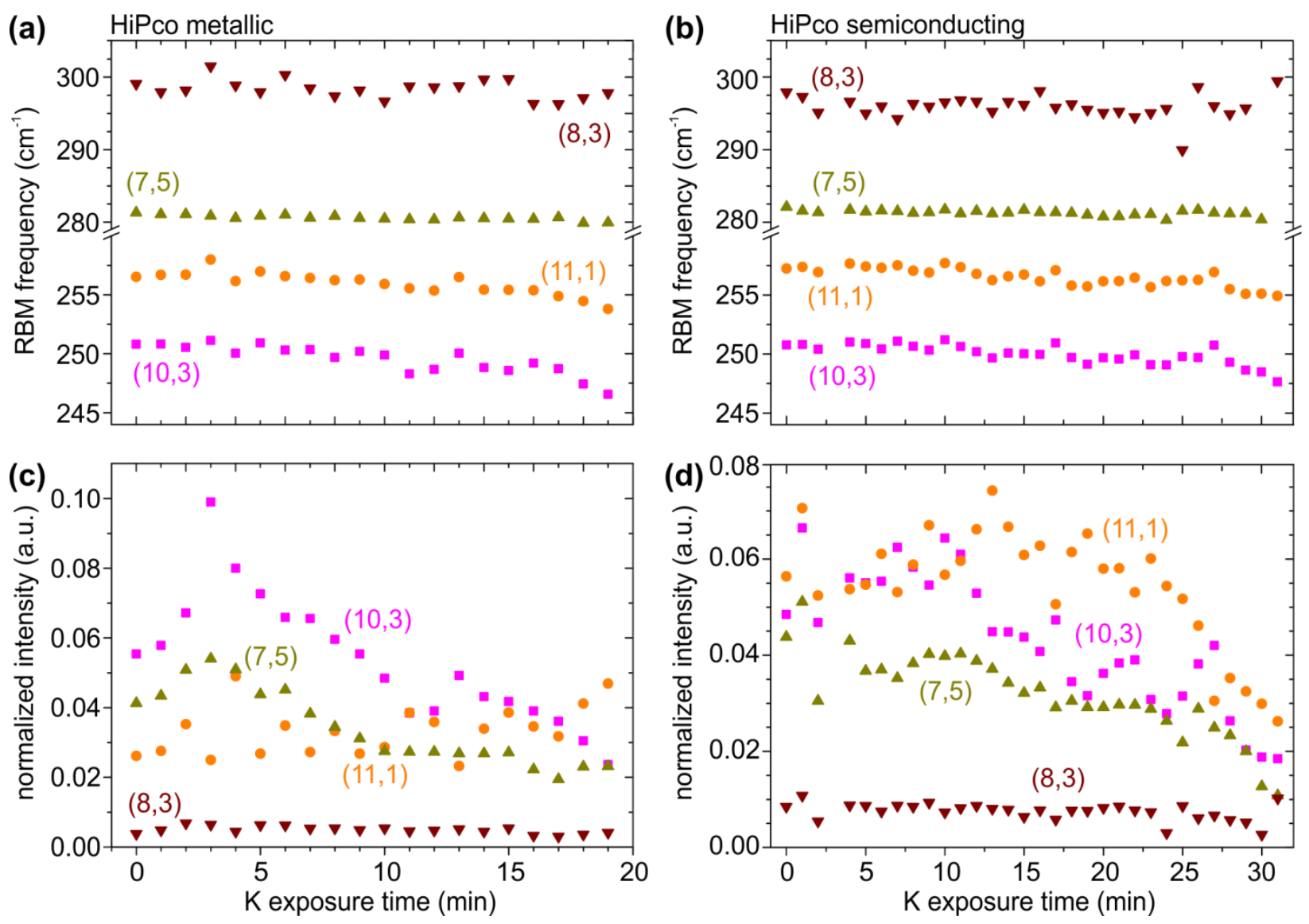

Figure S4. Evolution of the (a,b) RBM frequency and (c,d) normalized intensity for the (a,c) metallic and $(b, d)$ semiconducting enriched samples as a function of $\mathrm{K}$ exposure time for $(10,3)$ (magenta), (11,1) (orange), (7,5) (dark yellow), and $(8,3)$ (dark red) SWCNTs. The data is obtained from the fine-deconvolution of the RBM region of each recorded spectrum during the monitoring of the $\mathrm{K}$ doping. 
VII. RBM shift and intensity development of the metallic SWCNTs in the metallic enriched sample
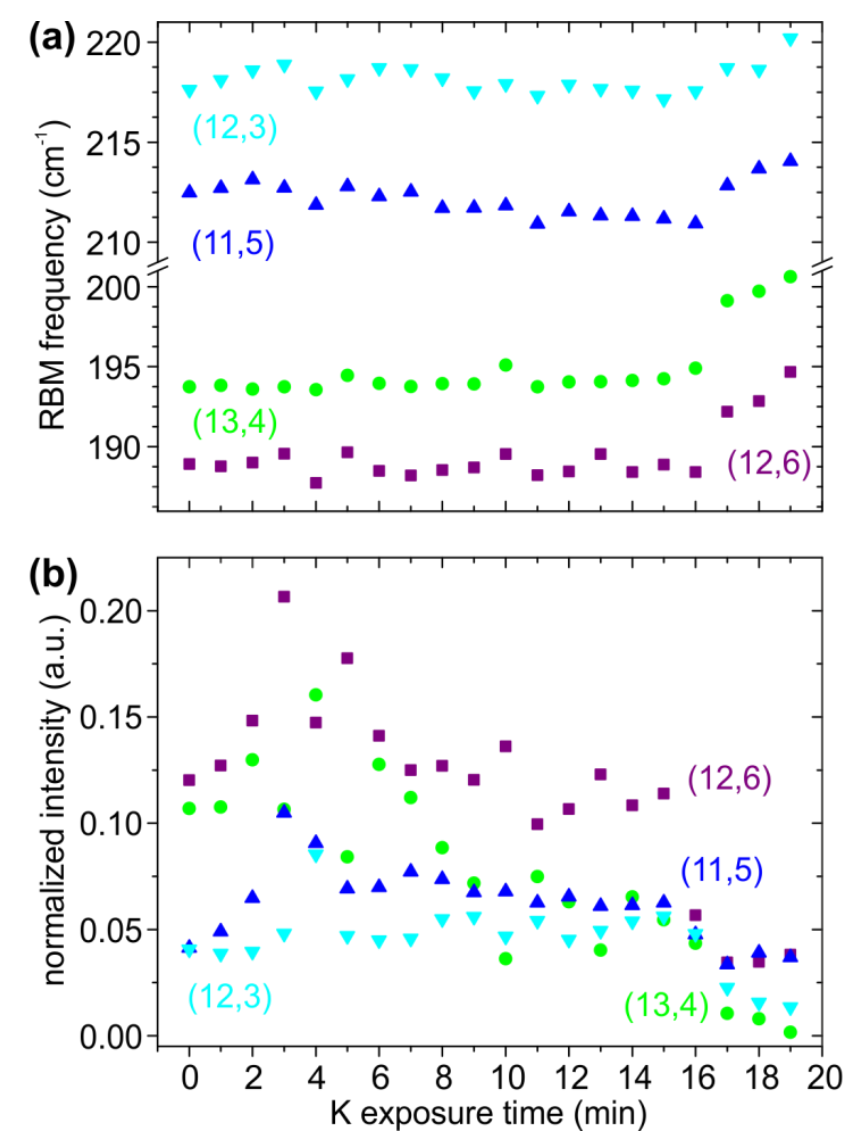

Figure S5. Evolution of the (a) RBM frequency and (b) normalized intensity as a function of $\mathrm{K}$ doping for the $(12,6)$ (violet), $(13,4)$ (green), $(11,5)$ (blue), and $(12,3)$ (cyan) SWCNTs in the metallic enriched sample. The data is obtained from the fine-deconvolution of the RBM region of each recorded spectrum during the monitoring of the $\mathrm{K}$ doping. 
VIII. Linear fitting functions of the calculated RBM frequency downshift for the $(8,4)$ SWCNT (Figure 4a)

\begin{tabular}{cc}
\hline section & \multicolumn{1}{c}{ linear fit function } \\
\hline 1 & $\omega_{1}=0.385 \mathrm{x}+0.092 \mathrm{~cm}^{-1}$ \\
\hline 2 & $\omega_{2}=1.830 \mathrm{x}+8.200 \mathrm{~cm}^{-1}$ \\
\hline 3 & $\omega_{3}=0.328 \mathrm{x}-1.680 \mathrm{~cm}^{-1}$ \\
\hline 4 & $\omega_{4}=1.464 \mathrm{x}+10.330 \mathrm{~cm}^{-1}$ \\
\hline 5 & $\omega_{5}=0.819 \mathrm{x}+2.360 \mathrm{~cm}^{-1}$ \\
\hline
\end{tabular}

Table S2. All linear functions obtained from the linear fits of the calculated shift of $(8,4)$ with increasing doping level.

IX. Linear fitting functions of the calculated RBM frequency downshift for the $(10,0)$ SWCNT (Figure 4b)

\begin{tabular}{cl}
\hline section & \multicolumn{1}{c}{ linear fit function } \\
\hline 1 & $\omega_{1}=0.346 \mathrm{x}+0.009 \mathrm{~cm}^{-1}$ \\
\hline 2 & $\omega_{2}=2.760 \mathrm{x}+16.300 \mathrm{~cm}^{-1}$ \\
\hline 3 & $\omega_{3}=-5.980 \mathrm{x}-0.192 \mathrm{~cm}^{-1}$ \\
\hline 4 & $\omega_{4}=3.720 \mathrm{x}+33.10 \mathrm{~cm}^{-1}$ \\
\hline 5 & $\omega_{5}=-0.024-7.240 \mathrm{~cm}^{-1}$ \\
\hline 6 & $\omega_{6}=3.600 \mathrm{x}+41.00 \mathrm{~cm}^{-1}$ \\
\hline 7 & $\omega_{7}=0.060 \mathrm{x}-9.233 \mathrm{~cm}^{-1}$ \\
\hline 8 & $\omega_{8}=1.260 \mathrm{x}+9.667 \mathrm{~cm}^{-1}$ \\
\hline
\end{tabular}

Table S3. All linear functions obtained from the linear fits of the calculated shift of $(10,0)$ with increasing doping level. 
X. Parameters of the cubic spline fit for the $(8,4)$ SWCNT relative Fermi shift to the CBM (Figure 4c)

\begin{tabular}{cccc}
\hline \multicolumn{5}{c}{$\mathbf{y}=\mathbf{A x}^{\mathbf{3}}+\mathbf{B x}^{2}+\mathbf{C x}+\mathbf{D}$} \\
\hline Parameter & $\mathbf{1}$ & $\mathbf{2}$ & $\mathbf{3}$ \\
\hline A & -0.014 & $-3.296 \mathrm{E}-4$ & $-8.052 \mathrm{E}-5$ \\
\hline B & -0.100 & -0.006 & -0.006 \\
\hline C & -0.284 & -0.070 & -0.156 \\
\hline D & -0.426 & -0.264 & -0.851 \\
\hline
\end{tabular}

Table S4. Cubic spline fit parameters of the relative position of the Fermi energy $\varepsilon_{\mathrm{F}}$ with increasing negative charges of $(8,4)$.

XI. Parameters of the cubic spline fit for the $(10,0) \mathrm{SWCNT}$ relative Fermi shift to the CBM (Figure 4d)

\begin{tabular}{ccc}
\hline \multicolumn{3}{c}{$\mathbf{y}=\mathbf{A x}^{3}+\mathbf{B x}^{2}+\mathbf{C x}+\mathbf{D}$} \\
\hline Parameter & $\mathbf{1}$ & $\mathbf{2}$ \\
\hline A & -0.020 & $-3.561 \mathrm{E}-5$ \\
\hline $\mathrm{B}$ & -0.120 & -0.002 \\
\hline $\mathrm{C}$ & -0.290 & -0.045 \\
\hline $\mathrm{D}$ & -0.427 & -0.235 \\
\hline
\end{tabular}

Table S5. Cubic spline fit parameters of the relative position of the Fermi energy $\varepsilon_{\mathrm{F}}$ with increasing negative charges of $(10,0)$. 


\section{Linear fittings of the RBM frequency downshifts in the semiconducting species}
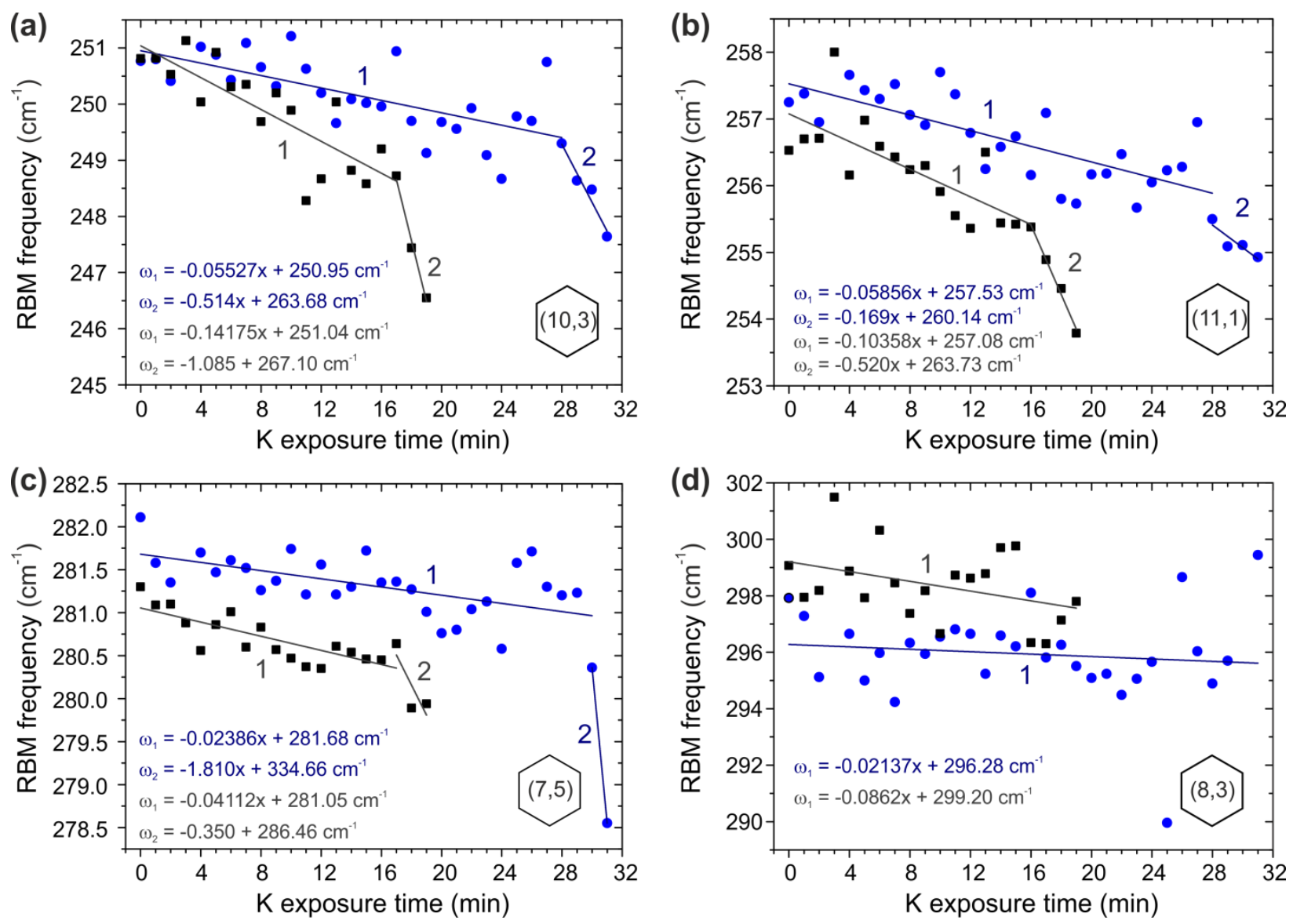

Figure S6. Linear fittings of the RBM development with increasing doping level of the semiconducting species found in our samples. (a) $(10,3)$, (b) $(11,1),(c)(7,5)$, and (d) $(8,3)$. The black dots and respective linear fits correspond to the metallic enriched sample and the blue spots and linear fits to the semiconducting enriched sample respectively. For each linear fit the corresponding function is given. Except of $(8,3)$ for all SWCNTs two linear fits were applied since the slope changed at a certain doping time. We assume this change to appear from the shift of the Fermi energy into the first van Hove singularity. 
XIII. Linear fittings of the RBM frequency downshifts in the metallic species
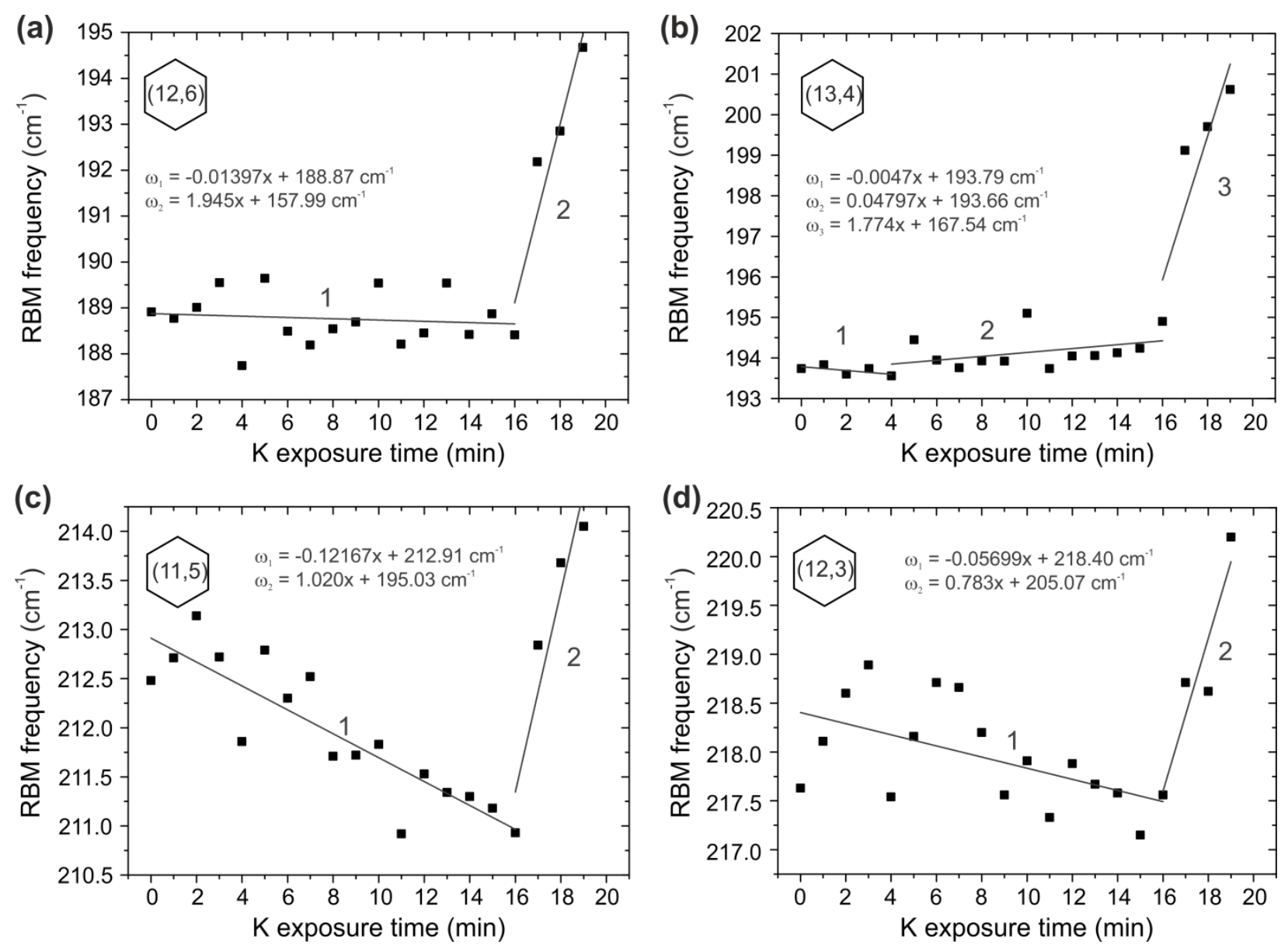

Figure S7. Linear fittings of the RBM development with increasing doping level of the metallic species found in the metallic enriched sample. (a) $(12,6)$, (b) $(13,4)$, (c) $(11,5)$, and (d) $(12,3)$. Separate linear fits were applied for the downshift and for the following upshift observed in the RBM frequency of the metallic species. 


\section{Calculation of the Free Energy $\Delta F^{0}$ and pressure of the potassium vapor phase in our samples}

To get comparable results from our experiment we calculated the potassium vapor pressure in the ampules regarding the following equation:

$$
-R \ln P=\Delta F^{0} / T
$$

where $\mathrm{P}$ is the pressure, $\mathrm{R}$ the Boltzmann constant $(=0.001987206 \mathrm{kcal} /(\mathrm{mol} \times \mathrm{K})$ and $\mathrm{T}$ the temperature.

To estimate the free energy $\Delta \mathrm{F}^{0}$ of potassium in our prepared sample ampules with heating we were fitting the values from reference [5] by a cubic fit (Figure S7). The heating temperature of the semiconducting enriched sample was $\sim 85^{\circ} \mathrm{C}(358.15 \mathrm{~K})$ and for the metallic enriched sample $\sim 90{ }^{\circ} \mathrm{C}(363.15 \mathrm{~K})$.

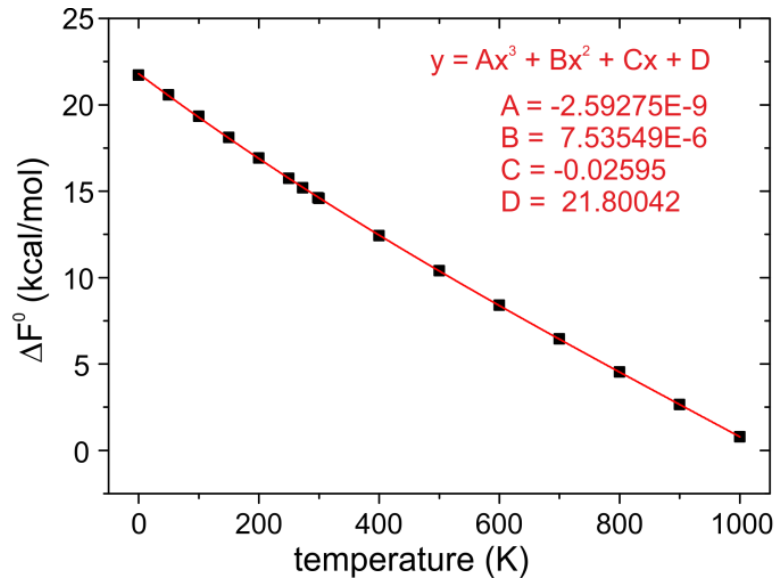

Figure S8. Cubic fit of the free energy of gaseous potassium dependent on its temperature from reference [10]. The received free energies at $358.15 \mathrm{~K}$ for the semiconducting enriched sample and $363.15 \mathrm{~K}$ for the metallic enriched sample are $13.354 \mathrm{kcal} / \mathrm{mol}$ and $13.246 \mathrm{kcal} / \mathrm{mol}$, respectively.

Rearranging equation (1) is leading to:

$$
P=e^{\left(\Delta F^{0} / R T\right)}
$$

The calculated potassium vapour pressure for the semiconducting enriched sample was 7.102E-09 atm (5.397 Torr) and for the metallic enriched sample 1.067E-08 atm (8.113 Torr). 


\section{References}

[1] J. Maultzsch, H. Telg. S. Reich, C. Thomsen, Phys. Rev. B 2005, 72, 205438.

[2] E. Menna, F. D. Negra, M. D. Fontana, Phys. Rev. B 2003, 68, 193412.

[3] F. Hennrich, R. Krupke, S. Lebedkin, K. Arnold, R. Fischer, D. E. Resasco, M. M. Kappes, J. Phys. Chem. B 2005, 109, 10567.

[4] H. Telg, J. Maultzsch, S. Reich, F. Hennrich, C. Thomsen, Phys. Rev. Lett. 2004, 93, 177401.

[5] W. H. Evans, R. Jacobson, T. R. Munson, D. D. Wagman, J. Res. Natl. Bur. Stand. (U.S.) 1955, 55, 83-96. 